Proceedings of the 2010 Winter Simulation Conference

B. Johansson, S. Jain, J. Montoya-Torres, J. Hugan, and E. Yücesan, eds.

\title{
AN EXPLORATION OF THE EFFECTS OF MAINTENANCE MANNING ON COMBAT MISSION READINESS UTILIZING AGENT BASED MODELING
}

\author{
Adam MacKenzie \\ J.O. Miller \\ Raymond Hill \\ Department of Operational Sciences \\ Air Force Institute of Technology \\ 2950 Hobson Way, Bldg 641 \\ Wright Patterson AFB, OH 45433 - 7765, USA
}

\begin{abstract}
Agent based models are powerful tools in describing processes and systems centered on individual behaviors and local interactions. Current application areas tend to be focused within the business and social science arenas, although their usefulness has been demonstrated in the modeling of various chemistry and physics-based systems and more recently, in unit-level military combat operations. Conversely, many highly process-oriented systems, such as manufacturing environments, tend to be modeled via "topdown" methods, including discrete or continuous event simulations. As a result, potentially critical attributes of the modeled entities or resources (spatial properties or adaptability) may not be adequately captured or developed. This research develops an agent based model for application to a problem heretofore addressed solely via discrete event simulation or stochastic mathematical models. Specifically, a model is constructed to investigate the effects of differing levels of maintenance manning on sortie production capability, while examining those effects on the resulting Combat Mission Readiness (CMR) of a typical F-16 squadron.
\end{abstract}

\section{INTRODUCTION}

In the summer of 2008, after noticing a highly variant trend in the combat mission readiness (CMR) of his assigned forces, the commander of United States Air Forces in Europe (USAFE) tasked his staff to examine all facets of the CMR process and determine what the major causal factors were (Lipina, 2009). As a result of this tasking, a regression study was completed in 2009 that quantified a variety of factors driving the variation in the CMR metric (Lipina, 2009). Based on the study's results, which were briefed to the Air Force Chief of Staff in the spring of 2009, additional taskings were levied on the operations (AF/A3) and logistics (AF/A4) directorates of the Air Staff. While AF/A3 embarked on an enterprise assessment of the CMR metric's composition, documentation and reporting requirements, AF/A4 was tasked to examine and address the use of aircraft maintenance capability metrics within the Air Force. Specifically, it was identified that no standard definition of maintenance capability existed, and the current methods and models used across the Air Force fail to adequately and convincingly capture the effects of maintenance capability on production capacity (AF/A4L, 2009).

There is a significant body of work that addresses multiple questions surrounding a maintenance unit's capacity and capabilities. This research revealed a string of studies dating back almost 30 years, all focused in some part on a typical maintenance unit's ability to successfully meet its operational requirements. A characteristic theme within all of these studies is a focus and evaluation of the units sortie generation ability. The specific objectives vary greatly, spanning everything from general system observa- 
tion and characterization to attempts to optimize one or more constituent sub-processes within the overall sortie generation process. These research efforts have employed many analytical methods, including discrete event simulation (Faas, 2003; Iakovidis, 2005), Markov decision analysis (Dietz, 1991) and neural networks (Dagg, 1991). Some of these research efforts even specifically addressed the issue of maintenance capability and its potential effects on sortie production and overall readiness (Gotz and Stanton, 1986; Garcia and Racher, 1981). Regardless, the methodologies utilized follow a more traditional approach of decomposing the system under investigation and attempting to describe its behavior as the "sum of its parts", which has been shown to be "inadequate to model and analyze" some large and complex systems (Kaegi et al, 2009). In fact, research performed across multiple disciplines has shown that these traditional methods of system decomposition and subsequent reconstitution can prove not only inadequate but also potentially produce misleading results (Bobashev et al, 2007). Kaegi et al (2009) further argue that in these situations, agent based modeling (ABM) has a "high potential to help realistically model large and complex systems".

Sortie generation involves an exceptionally complex set of processes with a variety of stochastic elements and external influences. Whether one contemplates the individual electrician or crew chief on the flight line, the supply troop in the Logistics Readiness Squadron, or even individual aircraft or parts being moved up and down the supply chain, each plays a fundamental role in the overall process. Additionally, while the behaviors and motivations of each of these constituent pieces is relatively well understood, the resulting behavior of the system as a whole is more complex than any explanation any individual component could provide. This is the hallmark of a complex system (Flake, 2002), an environment directly suited to an ABM's ability to reveal "properties of systems that are not properties of the agents themselves" (Jones, 2007).

\section{AN AGENT BASED SORTIE GENERATION SIMULATION}

A model of the sortie generation process benefits from use of an ABM structure due to its identified complexity. Looking specifically at the inter-relationships between maintenance personnel across a variety of skill levels and job specialties and their potential outputs in terms of sortie production, an ABM provides a detailed individual-based perspective on the overarching process. The specific focus of this research involves the on-equipment maintenance portion of the sortie generation process. The simulation model is used to examine the effects of various levels of maintenance manning on sortie production and manning utilization while taking into account the specific abilities of individual maintenance personnel across a variety of job specialties and skill levels.

\subsection{Simulation Model Development}

Development of the model required several key assumptions. These were: a) flying window remains constant; b) sortie load retains a set weekly pattern; c) aircraft configuration is not a concern; d) scheduled maintenance is not modeled; and e) collected data used to determine underlying distributions is assumed to be accurate and representative of the underlying real-world systems.

Figure 1 displays a top-level view of the modeled sortie generation process. Within the model, four separate agent types interact according to specific defined behaviors in order to accomplish the tasks making up various portions of the sortie generation process. In brief, the defined agent types are: Production Supervisor, Expediters, Aircraft and Maintenance agents. The Production supervisor provides general oversight and direction to the other agents. The expediter agents allocate personnel to their assigned tasks and are broken down into crew chief, avionics and mechanical (electro-environmental and propulsion) specialties. 


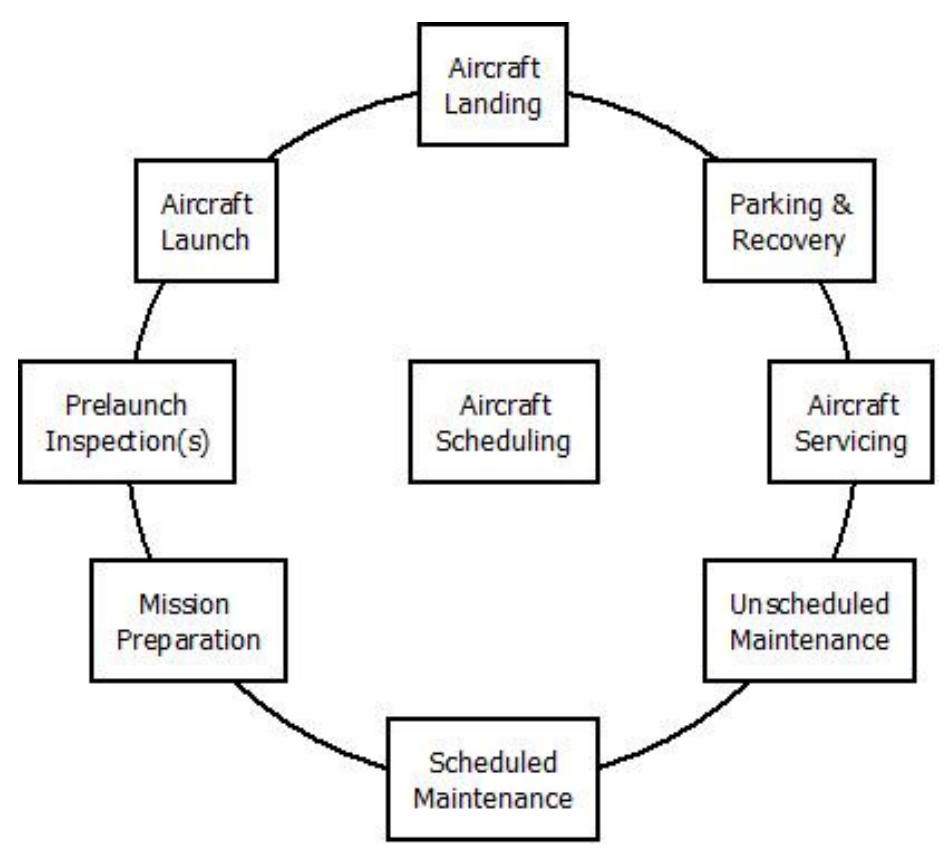

Figure 1: Modeled Sortie Generation Process

Finally, the maintenance agents serve as assignable resources and are further defined by their Air Force Specialty Code (AFSC) of crew chief, avionics, electro-environmental or propulsion; and skill level (3-, 5- or 7-level). Each of the maintenance agents possess a learning curve which models their increase in efficiency (modeled as working speed) over time.

\subsection{Model Execution}

The model begins in an empty and idle state. Following the general outline of the process depicted in Figure 1, a sortie requirement is placed on the system, which results in the assignment of specific aircraft to the sortie and an allocation of additional aircraft designated as spares. Maintenance jobs are assigned to each of these aircraft, which are in turn assigned to individual maintenance crews by the appropriate expediter. Depending on the priority assigned to each job by the pro super, some jobs are opened as training opportunities for junior (3-level) maintenance troops. As jobs are completed, the released aircraft will fly their assigned sorties, return and be prepared for follow-on missions or receive their end-of-day inspections. This cycle is continuously repeated, with sorties being scheduled 5 days a week, and maintenance crews operating on 3 rotating 8-hour shifts per day.

Aircraft breaks are stochastically determined at various points within the model. Each ground inspection carries a chance to uncover an issue, and each sortie has the potential for either an abort (immediate sortie failure) or Code 3 break (sortie completion, but aircraft lands with a maintenance issue). At each of these junctures, a random draw determines the number of issues found, and each of their associated Work Unit Codes (WUCs). All maintenance crew sizes and fix-time lengths for each task are stochastically determined via a multi-branch conditional tree. Conditioned on the identified WUC, separate trees identify both an AFSC and then a fitted theoretical distribution from which a fix-time is drawn. Finally, an assessment is made of the assigned team's mean efficiency rating, which is then used to modify the drawn fix time. The net effect is lengthened fix-times for lower efficiencies, with the unit's mean efficiency (and thus speed of work) increasing over time as individual maintenance agents learn their jobs. All repair distributions were based on 2 years of data from Spangdahlem AB, Germany. 


\section{ANALYSIS}

This section provides an analysis of the model's output when populated with a typical maintenance unit's maintenance manning levels. An additional response, maintenance efficiency, is also discussed.

\subsection{Experimental Design}

Fourteen core responses were identified as outputs of interest from the model. Twelve responses were the daily utilization rates of each AFSC and skill level, and the final two were the weekly figures for sorties produced and sortie cancellations. An additional set of responses, maintenance efficiencies, were also analyzed. Each agent has a maintenance efficiency varied from 0 (no skill) to 1 (highly skilled). Each agent also had a learning curve (defined by AFSC) that was used to increase their efficiency based upon experience (time spent on tasks in model). This measured the average increase in efficiency across each AFSC and skill level, capturing the effects of varied manning availability scenarios on the ability of a unit to continue to train and develop its junior maintenance troops. Four factors of interest were identified to drive the experiment: manning levels for each of the four AFSCs modeled. A full-factorial $2^{4}$ experiment was executed. Using a baseline level of manning modeled after data gathered from Shaw AFB as centerpoints, high and low test levels were formulated based on a $10 \%$ increase or reduction in available manning for each AFSC.

\subsection{Results}

Test results were based on a run length providing slightly over 7 months of operations and data after deleting the first week. While the majority of agent learning curves were based on a 12 month developmental cycle, this shorter time span allowed for additional replications and test points while still providing a solid indication of increased proficiency. After initial testing, it was determined that output variance stabilized after 20 replications; 25 replications per design point was used in subsequent analysis. Post-test evaluation of the responses indicated no severe departures from normality, so a series of standard ANOVA tests was performed for each of the responses. Results are provided in Table 1.

Table 1: Experiment Results

\begin{tabular}{|c|c|c|c|}
\hline Responses & $\begin{array}{l}\text { Significant } \\
\text { Factor(s) }\end{array}$ & Responses & Significant Factor(s) \\
\hline CC3 UTE & $\mathrm{CC}, \mathrm{AV}^{*}$ Manning & EE3 UTE & EE Manning \\
\hline CC5 UTE & CC Manning & EE5 UTE & $\begin{array}{c}\mathrm{CC}, \mathrm{AV}^{*}, \mathrm{EE}, \mathrm{JET} * \\
\text { Manning }\end{array}$ \\
\hline CC7 UTE & $\mathrm{CC}, \mathrm{AV}^{*}$ Manning & EE7 UTE & CC, AV, EE Manning \\
\hline AV3 UTE & AV Manning & JET 3 UTE & AV, JET* Manning \\
\hline AV5 UTE & AV, JET* Manning & JET 5 UTE & CC, JET Manning \\
\hline AV7 UTE & AV Manning & JET 7 UTE & CC* Manning \\
\hline Sorties/Week & AV Manning & Cancels/Week & AV Manning \\
\hline \multicolumn{4}{|c|}{$\begin{array}{l}\text { Legend: CC - Crew Chiefs, AV - Avionics, EE - Electro-Environmental, JET - Propulsion } \\
\text { UTE - Utilization } \\
* \text { - P-value for significance (F-test) greater than } 0.05 \text {, but less than } 0.1\end{array}$} \\
\hline
\end{tabular}

While the results presented in Table 1 are based on the varied numerical results provided from the model, the numbers are not the response of interest. With the variety of abstractions utilized to develop the model, the utility of these numerical responses as point estimators for true system performance is questionable. Instead, the surprising number of dependencies identified between disparate groups of manning is of key interest, chiefly because no interaction between any of these groups was included as a part of the model logic. 
Using the EE5 UTE response as an example, the original assumption was that the only factor of significance would be the EE manning pool. However, when one considers that the CC and AV AFSCs received the highest number of taskings, or that the average JET fix times tend to be somewhat lengthy, it is easy to see that modifications to the available manning for any of these AFSCs might drive maintenance timelines. Coupled with the fact that job priorities upgrade automatically if timelines were not being met, and potentially prohibit completion of any training, one can see that this could lead to shortened job times since the trainers (5- and 7-levels) are no longer slowing down work efforts in order to train lower skilllevel members.

A separate evaluation was performed on the effects on efficiency gains as a result of differing manning levels. Figure 2 depicts results from the extreme cases, comparing the baseline case to the reduction and addition of $10 \%$ manning for all AFSCs simultaneously. The results serve as a reminder of the additional complexities within the modeled system. In this case, the seemingly counterintuitive reduction in overall efficiency gains is actually a result of the additional manning added to shifts where work load was not as heavy. As such, these results are largely colored by circumstances peculiar to the simulation, but also underscore the importance of proper shift manning, in addition to the overarching import of sufficient manning levels unit-wide.

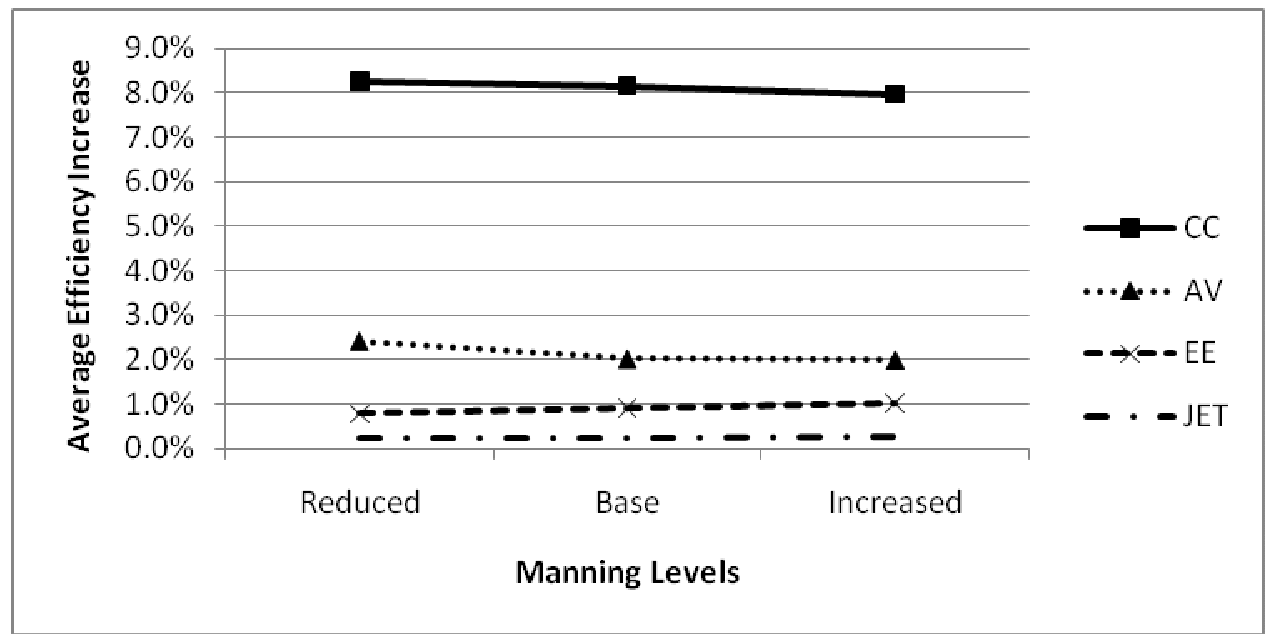

Figure 2: Comparison of Average 3-level Efficiency Increase

\section{CONCLUSIONS}

This model and analysis has examined the effects of varied levels of manning availability on both the utilization and production capacity of a maintenance unit. As previously mentioned it is not the intent to assign specific significance to the numerical results presented, but instead to the insight gained on the multiple layers of interactions between the various AFSCs and skill levels in executing the sortie generation process.

The results presented indicate a series of complex interactions between the various groups that make up the primary execution arm of the sortie generation process. Analysis shows that even slight changes in the structure of available manning can have significant effects on a unit's maintenance capacity and ability to develop over time. This has direct implications to any maintenance unit's capability to sustain and support a wing's CMR requirement.

\section{DISCLAIMER}

The views expressed in this article are those of the authors and do not reflect the official policy of the United States Air Force, the Department of Defense, of the US Government. 


\section{REFERENCES}

AF/A4L. 2009. Minutes of the Operational Maintenance Capability and Capacity Modeling (OMC2M) Workshop, Langley AFB, 15-17 December 2009.

Bobashev, G.V., D. M. Goedecke, F. Yu, and J. Epstein. 2007. "A Hybrid Epidemic Model: Combining the Advantages of Agent-based and Equation-based Approaches," In Proceedings of the 2007 Winter Simulation Conference, eds. S. G. Henderson, B. Biller, M.-H Hsieh, J. Shortle, J. D. Tew, and R. R. Barton, 1532-1537. Piscataway, New Jersey: Institute of Electrical and Electronics Engineers, Inc.

Dagg, J.M. 1991. An Exploratory Application of Neural Networks to the Sortie Generation Forecasting Problem. MS Thesis, AFIT/GLM/LSM/91S-11. School of Systems and Logistics, Air Force Institute of Technology (AU), Wright Patterson AFB OH, September 1991.

Dietz, D.C. 1991. Optimal Specialization and Allocation of Maintenance Manpower. PhD Dissertation. The Pennsylvania State University, University Park PA, 1991.

Faas, P.D. 2003. Simulation of Autonomic Logistics System (ALS) Sortie Generation. MS Thesis, AFIT/GOR/ENS/03-07. School of Engineering and Management, Air Force Institute of Technology (AU), Wright Patterson AFB OH, March 2003.

Flake, G.W. 2002. "The Computational Beauty of Nature." Excerpt from published book. http://mitpress.mit.edu/books/FLAOH/cbnhtml/parts.html. November, 2002.

Garcia, R., and J.P. Racher. 1981. An Investigation Into a Methodology to Incorporate Skill Level Effects Into the Logistics Composite Model. MS Thesis, AFIT/LSSR/81. School of Systems and Logistics, Air Force Institute of Technology (AU), Wright Patterson AFB OH, June 1981.

Gotz, G.A., and R.E. Stanton. Modeling the Contribution of Maintenance Manpower to Readiness and Sustainability. Contract MDA903-85-C-0030. Santa Monica, CA: RAND Corporation, January 1986.

Iakovidis, K. 2005. Comparing F-16 Maintenance Scheduling Philosophies. MS Thesis, AFIT/GLM/ENS/05-12. School of Engineering and Management, Air Force Institute of Technology (AU), Wright Patterson AFB OH, June 2005.

Jones, G.T. 2007. “Agent-based Modeling: Use with Necessary Caution.” American Journal of Public Health, 97(5): 780-781 (May 2007).

Kaegi, M., R. Mock, and W. Kroger. 2009. "Analyzing Maintenance Strategies by Agent-based Simulations: A Feasibility Study." Reliability Engineering and System Safety, 94(9): 1416-1421 (September 2009).

Lipina, A.J. 2009. Identifying Critical Factors Affecting Combat Mission Ready Status Among USAF Europe's Aircrew. Graduate Research Project, AFIT/IOA/ENC/09-01. School of Engineering and Management, Air Force Institute of Technology (AU), Wright Patterson AFB OH, June 2009.

\section{AUTHOR BIOGRAPHIES}

ADAM MACKENZIE is a Mar 2010 graduate of the Air Force Institute of Technology, receiving a MS in Operations Research. After graduate he was assigned as an analyst at AF/A9. He can be reached at <Adam.Mackenzie@pentagon.af.mil>.

J. O. MILLER is a 1980 graduate of the U.S. Air Force Academy (USAFA) and retired from the Air Force as a Lt. Colonel in January 2003. In addition to his undergraduate degree from USAFA, he received an MBA from the University of Missouri at Columbia in 1983, his M.S. in Operations Research from the Air Force Institute of Technology (AFIT) in 1987, and his Ph.D. in Industrial Engineering from The Ohio State University in 1997. Dr. Miller is Director of the Center for Operational Analysis and an associate professor in the Department of Operational Sciences at AFIT. His research interests include combat modeling, computer simulation, and ranking and selection. He can be reached at

<john.millerdafit.edu> . 
RAYMOND R. HILL is an Assistant Professor of Operations Research in the Department of Operational Sciences at the Air Force Institute of Technology. His research interests include modeling and simulation, applications of optimization, and military applications of modeling. He holds a Ph.D. from The Ohio State University. He can be reached at <raymond.hilleafit.edu> . 